\title{
ORIGINAL
}

\section{ENFERMEDADES INFECCIOSAS Y CARACTERÍSTICAS SOCIODEMOGRÁFICAS DE LOS INMIGRANTES EXTRANJEROS DEL CENTRO PENITENCIARIO DE HOMBRES DE BARCELONA}

\author{
Joan García Vidal (1), Josep M. Jansà López del Vallado (2), Patricia García de Olalla Rizo (2), \\ Isabel Barnés Vayés (1), Joan A. Caylá Buqueras (2). \\ (1) Centro Penitenciario de Hombres Barcelonia. Departamento de Justícia-Generalitat de Catalunya. \\ (2) Servicio de Epidemiología. Instituto Municipal de la Salud de Barcelona. Unidad de Investigación en Tubercu- \\ losis de Barcelona
}

\section{RESUMEN}

Fundamento: La inmigración de países de bajo desarrollo socio-económico es relativamente reciente en nuestro país. Analizar el impacto de enfermedades como el VIH/sida y la tuberculosis en estas poblaciones, presenta especial interés en materia de salud pública.

Métodos: Estudio de casos y controles, realizado en el Centro Penitenciario de Hombres de Barcelonit, entre el 1/01 y el $30 / 09$ de 1996. siendo los casos los inmigrantes extranjeros de países de bajo desarrollo socio-económico. y los controles los internos autóctonos apareados por edad $(+f-5$ años $)$. Se describen las características epidemiológicas y sociodemográficas de los inmigrantes, y se comparan ambas poblaciones.

Resultados: Se incluyeron 450 internos. 300 autóctonos y 150 inmigrantes. El $63 \%$ de los inmigrantes eran masrebíes, un $72 \%$ se hallaban en situación irregular, el 55\% llevaban más de 5 años de residencia en nuestro país y el $53 \%$ no disponía de convivencia familiar. Los inmigrantes consumían menos drogas por vía parenteral que los autóctonos (OR: 0.2 : IC $95 \% 0,09-0,41$ ), presentaban menos infección VIH (OR:0,2: IC $95 \% 0.05-0.49)$ y vivían menos en familia (OR: 0.2; $\mathrm{IC}_{95}, 0.11-0.36$ ). No había diferencias en cuanto a infección por Mycobacteritun tuberculosis ni a enfermedad tuberculosa. Los inmigrantes regularizados eran mayores (OR:1,07: IC45\% 1,01-1.13). convivían más en familia (OR:2.7: IC $95 \% 1.23-5.80)$ y consumían más alcohol (OR:1.7: IC $95 \% 1,07$ 2.59) que los no regularizados.

Conclusiones: Los internos autóctonos estiban más afectados por el VIH que los inmigrantes, influyendo el uso parenteral de drogas, más frecuente entre los autóctonos. En los inmigrantes la elevada frecuencia de situacione: no regularizadas y la ausencia de núcleo familiar estable constituyen indicadores de marginación social.

Palabras Clave: Inmigrante, prisión, VIH, SIDA, tuberculosis, enfermedades infecciosas, alcohol, drogas.

Correspondencia:

Josep María Jansà

Servicio de Epidemiología

Institut Municipal de la Salut

Plaça Lesseps 1

08023 Barcelona

Tel: 934150066 Fax: 932182275

Correo electrónico: jjansa@imsb.bcn.es

\section{ABSTRACT}

\section{Infectious Diseases and Characteristics of the Foreign Immigrants at the Male Prison Facility in Barcelona}

Background: Immigration from socioeconomically underdeveloped countries is relatively recent in our country. Analyzing the impact of diseases such as HIV/AIDS and tuberculosis among these populations is of particular interest with regard to public health.

Methods: Case study and tests conducted at the Male Prison Facility in Barcelona from January 1, 1996 to September 30, 1996 , the cases being the foreign immigrants from socioeconomically underdeveloped countries, and the checks of the native prisoners paired by age $(+/-5$ years). A description is provided of the epidemiological and sociodemographic characteristics of the immigrants, and a comparison is drawn between the two populations.

Results: 450 prisoners, 300 native and 150 immigrants. Sixly-three percent $(63 \%)$ of the immigrants were from the Maghreb, $72 \%$ being in this country illegally, $55 \%$ having resided in our country for over 5 years and $53 \%$ not living with any family members. The immigrants used less injected drugs that the native prisoners (OR: 0.2; IC $95 \%$ 0.09-0.41), had less HIV infection (OR: 0.2; IC $95 \%$ 0.05-0.49) and lived to a lesser extent with family (OR: 0.2 : IC $95 \% 1.011-0.36$ ). No differences were found to exist with regard to infection by Mycobacterium iuberculosis nor tubercular disease. The legal immigrants were older (OR: 1.07; IC $95 \%$ 1.01-1.13) lived to a greater extent with family (OR: $2.7 ; \mathrm{IC}_{05} \% 1.23-5.80$ ) and drank more alcohol (OR: 1.7; $\mathrm{IC}_{05 \%}$ (.07-2.59) than the illegal residents.

Conclusions: The native prisoners had HIV to a greater extent than the immigrants, the use of injected drugs, more common among the natives, bearing an influence on this aspect. As regards the immigrants, the high degree of illegal residence and the absence of a stable core family comprise indicators of a situation of being socially disadvantaged.

Key Words: Immigrant. Prison. HIV. AIDS. Tuberculosis. Infectious diseases. Alcohol. Drugs. 


\section{INTRODUCCIÓN}

La migración de poblaciones humanas es un fenómeno tan antiguo como el deseo del hombre de mejorar. Se calcula que entre 1890 y 1990, 6,7 millones de españoles han emigrado a América, un millón y medio a países europeos, y un millón al continente africano. En la actualidad, el proceso migratorio de los españoles hacia el exterior por causas econóninicas es prácticamente insignificante, siendo en nuestros días el estado español un país receptor de inmigración ${ }^{1}, \mathrm{y}$ aunque la tendencia migratoria se haya invertido, el impacto global de los inmigrantes extranjeros en nuestro país sigue siendo muy discreto. Este hecho se aprecia en términos cuantitativos en el caso de la ciudad de Barcelona donde, asumiendo incluso la existencia de «poblaciones ocultas», los inmigrantes extranjeros representan solamente alrededor de un 3\% de la población, cifra que se reduce a menos de un $2 \%$ cuando se consideran únicamente a las personas procedentes de áreas de bajo desarrollo social y económico ${ }^{2}$.En los últimos años se han llevado a cabo estudios sociológicos, antropológicos y demográficos, que constituyen una aproximación a las características de este fenómeno $o^{3}$. Desde una óptica sanitaria, se han realizado aproximaciones y estudios en distintos ámbitos; salud materno-infantil $^{4}$, incidencia de sida y tuberculosis ${ }^{5}$ (TB), utilización y acceso a los recursos sanitarios, análisis de mortalidad y nacimientos de hijos de madres inmigradas ${ }^{6}$, por destacar algunos de los aspectos más relevantes.

A pesar de todo, quedan todavía distintas preguntas por responder en relación a cuál es la prevalencia e incidencia en estas poblaciones de algunas de las enfermedades infecciosas de mayor gravedad y frecuencia en nuestro medio. Para contribuir a dar respuesta a estos interrogantes, se llevó a cabo el presente estudio seroepidemiológico, en el que comparando poblaciones locales e inmigradas, se pretenden identificar algunos indicadores de salud de los inmigrantes ex- tranjeros procedentes de zonas socio-económicamente deprimidas.

El Centro Penitenciario de Hombres de Barcelona (CPHB), reúne diversas características que lo hacen especialmente idóneo para la realización de un estudio como el que se presenta, por un lado, porque al tratarse de una institución que acoge a un elevado número de individuos, permite la obtención de una muestra de población con relativa facilidad y, por otro lado, porque de los cerca de 2000 internos de esta institución penitenciaria, existentes diariamente durante el año 1996, un 18\% eran inmigrantes, de los cuales el $74 \%$ procedían de países de bajo desarrollo social y económico ${ }^{7.8}$. El que la mayoría de internos autóctonos procedan de niveles socioeconómicos menos favorecidos, contribuye a establecer posibles comparaciones con los inmigrantes que pueden estar sujetos a condiciones de marginalidad en los países de acogida.

Los objetivos de este estudio, se centran en identificar las características diferenciales socio-demográficas, de consumo de alcohol y drogas y los perfiles sero-epidemiológicos de patologías infecciosas, en la población inmigrada procedente de zonas de bajo desarrollo social y económico que ingresa en el CPHB, comparando dichos datos con los de la población de autóctonos en el mismo centro.

\section{SUJETOS Y MÉTODOS}

Se trata de un estudio de casos y controles en el que se compara la población inmigrada con la población local ingresadas en el CPHB entre los meses de enero y septiembre de 1996. Se consideró caso a cualquier individuo procedente de àreas de bajo desarrollo social y económico (África excepto la República Sudafricana, Centro y Sudamérica, Asia excepto Japón). Los inmigrantes extranjeros fueron agrupados en función de las categorías descritas por el Banco Mundial que han sido utilizadas en otros cstudios ${ }^{9}$. Sc 
seleccionaron dos controles por cada caso entre los internos autóctonos que ingresaban de forma correlativa a un inmigrante (los dos siguientes), con un intervalo de \pm cinco años de edad, para evitar posibles diferencias debidas a este factor en la medida que, mientras que los inmigrantes tienden a ser una población esencialmente joven, existe la posibilidad de que se dé una mayor variabilidad entre los autóctonos.

La selección de los casos y los controles se llevó a cabo en días alternos durante la semana, hasta conseguir 150 casos y 300 controles. El médico y la enfermera responsables del estudio en el servicio médico del $\mathrm{CPHB}$, realizaron una entrevista personalizada a cada caso y a cada control en el momento de ingresar en el centro. La entrevista se basaba en una encuesta en la que se recogían datos de filiación, tiempo de residencia en el estado español, nivel de conocimiento del castellano (excluyendo a los de América Latina), tipo de convivencia domiciliaria, consumo de alcohol ${ }^{10}$, drogas (considerando los patrones de consumo parenteral, no parenteral y mixto para heroína y cocaína) y conductas de riesgo para la infección por VIH. Los datos sobre antecedentes de TB y diagnósticos de sida (enfermedad indicativa, fecha y lugar de diagnóstico), y hepatitis virales, con posterioridad a la llegada al estado español en el caso de los inmigrantes, se obtuvieron a partir de la revisión de la documentación clínica de los encuestados. Así mismo, en el momento de ingresar en el CPHB, se realizó un análisis de sangre con determinación de serología específica para hepatitis víricas (IgM VHA, AcVHBc, AcVHBs, Ag VHBs, Ac VHC), lúes (RPR, VRDL), considerándose como válidos los resultados negativos documentados que se hubieran practicado en los seis meses previos al ingreso en el CPHB, y los positivos sin límite de tiempo.

Para la hepatitis B se consideraron como positivos a efectos del estudio, los individuos con resultados positivos a los marcadores AcVHBc y Ag VHBs, toda vez que se dispuso de los datos de los tres indicadores para todos los individuos de los que se obtuvo información sobre esta enfermedad.

Para la detección de la infección por VIH, se utilizaron las pruebas de ELISA y Western blott con el mismo criterio de validez. Los resultados positivos sobre VIH se obtuvieron en todos los inmigrantes de las analíticas practicadas en el estudio y sólo en 17 de los autóctonos $(13,4 \%)$ procedieron de la documentación clínica previa, mientras que para lúes $\mathrm{y}$ hepatitis $\mathrm{B}$, toda la información de resultados positivos fue obtenida de las serologías practicadas durante el estudio. Para detectar la infección tuberculosa se practicó la prueba de la tuberculina mediante la técnica de la intradermoreacción de Mantoux (IDR), con 2 Unidades Internacionales de PPD RT-23. No se pudo recoger información sobre los antecedentes vacunales de BCG, debido a dificultades en la interpretación de la escara vacunal en muchos de los inmigrantes. Se consideró como infectados por Mycobacterium tuberculosis a los individuos con una induración tuberculínica superior o igual a $5 \mathrm{~mm}$, aunque se valoró considerar la positividad en inmigrantes extranjeros a partir de $14 \mathrm{~mm}$ por su mayor probabilidad de haber sido vacunados con BCG. Existió una elevada proporción de resultados desconocidos en los valores serológicos (entre el 18 y el $45 \%$ ), especialmente entre los inmigrantes, debido a que, por ser el CPHB un centro de preventivos, ello determina que en muchas ocasiones los internos presenten estancias muy breves sin que, a pesar de su indicación, se llegara a tiempo de practicárseles la analítica correspondiente.

Los datos fueron recogidos en una encuesta específicamente diseñada al efecto y validada anteriormente, mediante una prueba piloto llevada a cabo antes del inicio del estudio. Para el análisis estadístico se utilizó el paquete de programas SPSS. A nivel bivariado se aplicaron las pruebas de ji cuadrado y la prueba exacta de Fisher y la t de Student para la comparación de medias, calculándo- 
se los Odds Ratios (OR) con intervalos de confianza del $_{95 \%}$ (IC). En el estudio descriptivo se realizó un análisis crudo de los datos, y a nivel multivariado se utilizó un modelo de regresión logística condicional, con las variables con significación estadística a nivel bivariado que se consideraron epidemiológicamente relevantes a efectos del estudio, utilizándose el sistema de exclusión de secuencias (Forward Stepwise), que introduce cada variable en función de su máxima verosimilitud. Para las variables edad, región de origen, situación administrativa, tiempo de residencia, nivel de castellano y consumo de alcohol, la proporción de desconocidos fue del $0 \%$; para las variables conducta de riesgo hom osexual, hemofílico - hemotransfundido, relación con prostitutas, tatuajes y tipo de convivencia familiar, se situaron entre el 0,5 y el $6 \%$, la inclusión en programas libres de drogas o PMM, la prueba de Mantoux y los antecedentes de TB, presentaron entre el 6,5\% y el $15 \%$ de valores desconocidos, toda vez que para los $\mathrm{AC}$ VHA, $\mathrm{AC}$ VHB, $\mathrm{AC}$ VHC, serología luética, VIH y sida esta cifra fue superior al $15 \%$. Se realizó un análisis de las características de los valores desconocidos frente a la variable clependiente, observándose que no existían diferencias en relación a los casos en los que se disponía de la información, lo que garantiza la validez de los resultados del análisis multivariante.

\section{RESULTADOS}

Se incluyeron 150 inrnigrantes extranjeros procedentes de áreas de bajo desarrollo social y económico y 3010 controles autóctonos, originarios en su mayor parte de $\mathrm{Ca}-$ talunya (63\%). Entre los inmigrantes extranjeros, la zona de procedencia más frecuente fue el Norte de África con un $63 \%$, destacando en primer lugar Argelia que, con 63 internos, representa el $42 \%$ del total de inmigrantes del estudio, seguido de 22 marroquíes $(15 \%)$ y 16 colombianos $(11 \%)$, presentando el resto de países proporciones muy inferiores (tabla 1). La edad media de los casos y de los controles fue de 30 años, desviación estándar (DE) 6,8 y 6,6 respectivamente, destacando a nivel sociodemográfico que un $72 \%$ de los inmigrantes se hallaban en situación administrativa irregular, a pesar de que un $55 \%$ de ellos llevaba más de cinco años residiendo en nuestro país.

En relación al consumo de sustancias un $79 \%$ de los inmigrantes extranjeros acreditó una ingesta regular de alcohol (más de 8 gramos/día). En cuanto al consumo de drogas ilegales, un $46 \%$ declararon fumar cannabis, el $18 \%$ de los cocainómanos y el $22 \%$ de los heroinómanos no utilizaba la vía parenteral, mientras que solamente $5,3 \%$ de los cocainómanos y $6,7 \%$ de los heroinómanos declararon utilizar solamente la vía parenteral para su consumo y $0,7 \%$ y $4,7 \%$ respectivamente acreditaban consumos mixtos (parenteral y no parenteral). En cuanto a la infección por $M$. Tuberculosis, la prevalencia en ambas poblaciones, y la de la infección por VIH se muestran en tabla 3 .

Al comparar los datos de los inmigrantes con las de sus controles autóctonos, a nivel bivariado, destaca el mayor porcentaje de tuberculín positivos entre los inmigrantes, sin que esta diferencia llegara a ser significativa. En cuanto al VIH, sin embargo, los niveles de infección fueron inferiores entre los inmigrantes $(\mathrm{OR}=0.1$ IC: $0,02-0,13)$, diferencia que se mantuvo en el análisis multivariado. Entre los inmigrantes, de los 5 reclusos VIH positivos identificados, 3 estaban coinfectados con $M$. Tuberculosis, significando una prevalencia de infección del $3 \%$, mientras que en los autóctonos la coinfección se identificó en el $28 \%$, coincidiendo esencialmente con los individuos usuarios de drogas por vía parenteral (UDVP).

En cuanto a los hábitos tóxicos, también a nivel hivariado, destaca, entre los que referían una historia de consumo de alcohol, el hecho de que los inmigrantes presentaban una media de 10,1 años de consumo (DE $5,9)$, frente a los 13,2 años (DE 6,7) de los 
Tabla 1

Características socio-demográficas de los ingresos en el cphb 01-01-96 y el 30-09-96. comparación inmigrantes / autóctonos

\begin{tabular}{|c|c|c|c|}
\hline & $\begin{array}{c}\text { Inmigrantes } \\
n=150(\%)\end{array}$ & $\begin{array}{l}\text { Autóctonos } \\
n=300(\%)\end{array}$ & $\begin{array}{c}\text { OR }(95 \% \text { IC) } \\
\text { o valor } p\end{array}$ \\
\hline Edad $\times(D E)^{*}$ & $29,9(6,8)$ & $30,4(6.6)$ & 0,6 \\
\hline Región de origen & & & - \\
\hline Catalunya & - & $188(62,7)$ & \\
\hline Resto del Estado Español & - & $112(37.3)$ & \\
\hline Norte de África & $93(62,7)$ & - & \\
\hline Latino-América y Caribe & $25(16.7)$ & - & \\
\hline Africa subsahariana & $21(14,0)$ & - & \\
\hline Extremo oriente y China & $11(07,3)$ & - & \\
\hline Con residencia o visado & & $42(28)$ & - \\
\hline Sin papeles & $108(72)$ & - & \\
\hline \multicolumn{4}{|l|}{ Convivencia familiar } \\
\hline en familia & $71(47,3)$ & $245(81,7)$ & $0,2(0,11-0,29)$ \\
\hline solo o varios & $79(52,7)$ & $49(16,3)$ & 1 \\
\hline desconocido & - & $6(2,0)$ & \\
\hline Tiempo de residencia & & & - \\
\hline hasta 6 meses & $17(11,3)$ & - & \\
\hline de 6 meses a 2 años & $19(12,7)$ & - & \\
\hline de 2 a 5 años & $32(21,3)$ & - & \\
\hline de 5 a 10 años & $62(41,3)$ & - & \\
\hline más de 10 años & $20(13,3)$ & - & \\
\hline \multicolumn{4}{|l|}{ Nivel de Castellano** } \\
\hline no entiende & $15(12,0)$ & $\ldots$ & \\
\hline entiende & $18(14,4)$ & - & - \\
\hline habla & $37(29,6)$ & - & \\
\hline lee & $18(14,4)$ & - & \\
\hline escribe & $37(29,6)$ & & \\
\hline
\end{tabular}

* Media y desviación estándar

** Se excluyen los procedentes de América-Latina (no hahía ningún caso de Brasil).

autóctonos, diferencia que era estadísticamente significativa $(\mathrm{p}=0,002)$. Así mismo, los inmigrantes reflejaban un consumo de drogas por vía parenteral significativamente inferior al de los autóctonos (p,001), toda vez que entre los heroinómanos los inmigrantes no utilizaban la vía parenteral con más frecuencia que los autóctonos $(\mathrm{p}, 05)$, al tiempo que, tanto los consumidores de cocaína como los de heroína entre los autóctonos, presentaban períodos de consumo significativamente superiores a los que presentaban los inmigrantes (tabla 2) Del mismo modo, mientras que sólo se identificó un caso de sida entre los inmigrantes extranjeros, se diagnosticaron 15 entre los autóctonos, diferencia que también era significativa a nivel bivariado $\mathrm{CR}=13$ (IC ${ }_{95 \%} 0,01-0,93$ ) $(\mathrm{p}=0.01)($ tabla 3$)$.

Los datos seroepidemiológicos sobre hepatitits víricas, a pesar del elevado número de desconocidos, reflejan una mayor proporción de infecciones por los virus $\mathrm{C}$ y $\mathrm{B}$ entre los autóctonos que en los inmigrantes (tabla 3).Al categorizar a estos últimos en función del tiempo de residencia en nuestro país (más o menos de cinco años), no se observó 
Tabla 2

Consumo de alcohol, drogas y factores de riesgo para el VIH comparación entre reclusos inmigrantes y autóctonos

\begin{tabular}{|c|c|c|c|c|}
\hline & & $\begin{array}{c}\text { Inmigrantes } \\
n=150(\%)\end{array}$ & $\begin{array}{l}\text { Autóctonos } \\
n=300(\%)\end{array}$ & $\begin{array}{c}\text { OR }(95 \% \text { IC) } \\
\text { o valor } p\end{array}$ \\
\hline Consumo alcohol & $\begin{array}{l}\text { sí } \\
\text { no }\end{array}$ & $\begin{array}{r}119(79,3) \\
31(20,7)\end{array}$ & $\begin{array}{r}236(78,7) \\
64(21,3) \\
\end{array}$ & $1,1(0,631,74) 1$ \\
\hline Consumo cannabis & $\begin{array}{l}\text { sí } \\
\text { no }\end{array}$ & $\begin{array}{c}69(46,0) 81 \\
(54,0) \\
\end{array}$ & $\begin{array}{r}202(61,7) \\
95(37,3) \\
\end{array}$ & $2,5(1.64-3,81) 1$ \\
\hline Consumo cocaína & $\begin{array}{l}\text { sí } \\
\text { no }\end{array}$ & $\begin{array}{r}36(24) \\
114(76) \\
\end{array}$ & $\begin{array}{l}185(61,6) \\
112(37,3) \\
\end{array}$ & $0,2(0,12-0,30) 1$ \\
\hline Consumo heroína & $\begin{array}{l}\text { sí } \\
\text { no }\end{array}$ & $\begin{array}{c}50(33,3) \\
100(66,7)\end{array}$ & $\begin{array}{c}200(66,7) \\
97(3,2)\end{array}$ & $0,2(0,15-0.37) 1$ \\
\hline Años consumo * alcohol (DE) & & $\begin{array}{c}10,0(5,7) \\
n=63\end{array}$ & $\begin{array}{c}13,2(6,7) \\
n=98\end{array}$ & 0,002 \\
\hline Años consumo * cannabis (DE) & & $\begin{array}{c}10,5(6,9) \\
\mathrm{n}=67\end{array}$ & $\begin{array}{c}10,3(5,4) \\
\mathrm{n}=198\end{array}$ & 0,809 \\
\hline Años consumo * cocaína (DE) & & $\begin{array}{c}3,4(3,26) \\
n=33\end{array}$ & $\begin{array}{c}6,0(4,7) \\
n=183\end{array}$ & 0,003 \\
\hline Años consumo * heroína (DE) & & $\begin{array}{c}4,0(3,1) \\
n=48\end{array}$ & $\begin{array}{c}7,8(4,6) \\
n=199\end{array}$ & 0,0005 \\
\hline UDVP & $\begin{array}{l}\text { sí } \\
\text { no }\end{array}$ & $\begin{array}{c}18(12) \\
132(88)\end{array}$ & $\begin{array}{l}184(61,3) \\
116(38,7) \\
\end{array}$ & $0.1(0.05-0.15) \mathrm{l}$ \\
\hline Homosexual (HMS) & $\begin{array}{l}\text { sí } \\
\text { no }\end{array}$ & $\begin{array}{c}1(0,7) \\
149(99,3)\end{array}$ & $\begin{array}{c}9(3,0) \\
288(96,0)\end{array}$ & $0.2(0,01-1,67)$ \\
\hline Hemofílico-hemotransfundido & $\begin{array}{l}\text { sí } \\
\text { no }\end{array}$ & $\begin{array}{c}6(4,0) \\
144(96,0) \\
\end{array}$ & $\begin{array}{c}24(8,0) \\
269(89,7) \\
\end{array}$ & $0,5(0,17-1,24) 1$ \\
\hline Relación prostitutas & $\begin{array}{l}\text { sí } \\
\text { no }\end{array}$ & $\begin{array}{l}55(36,7) \\
94(62,7)\end{array}$ & $\begin{array}{l}142,(47,3) \\
155(51,7)\end{array}$ & $0,6(0,42-0,97) 1$ \\
\hline Tatuajes & $\begin{array}{l}\text { sí } \\
\text { no }\end{array}$ & $\begin{array}{l}46(30.7) \\
99(66,0)\end{array}$ & $\begin{array}{c}202(67,3) \\
92(30,7)\end{array}$ & $0,2(0,13-0,33) 1$ \\
\hline Si UDVP en PLD** & $\begin{array}{l}\text { sí } \\
\text { no }\end{array}$ & $\begin{array}{c}3(16,7) \\
12(66,7) \\
\end{array}$ & $\begin{array}{l}87(47,3) \\
90(48,8) \\
\end{array}$ & $0,3(0,07-1,03) 1$ \\
\hline Si UDVP en PMM & $\begin{array}{l}\text { sí } \\
\text { no }\end{array}$ & $\begin{array}{c}1(5,6) \\
14(77,8)\end{array}$ & $\begin{array}{l}66(35,9) \\
111(60,3)\end{array}$ & $0,1(0,01-0,91) 1$ \\
\hline
\end{tabular}

* Media aritmética y desviación estándar entre paréntesis

** Programa libre de drogas.

que quienes residían desde hace más tiempo, presentaran una mayor frecuencia de situaciones administrativas regularizadas. A nivel sociodemográfico se observó cómo aquellos que emigraron con anterioridad (más de 5 años) tendían más a vivir en familia que los de emigración reciente (menos de 5 años), $\mathrm{OR}=0,4$ (IC $\left.{ }_{95 \%} 0,21-0,87\right)$. En relación al tiempo de emigración, no se observan diferencias en las variables tuberculín+, 
Tabla 3

Características sero-epidemiológicas. comparación entre inmigrantes y autóctonos a nivel bivariado

\begin{tabular}{|c|c|c|c|}
\hline & Innigrante (\%) & Autóctono (\%) & OR $(I C 95 \%)$ \\
\hline \multicolumn{4}{|l|}{$\mathrm{PPD}^{*}$} \\
\hline positivo & $76(50.7)$ & $133(44,3)$ & $1,3(0.82-1.95)$ \\
\hline negativo & $61(40,7)$ & $135(45,0)$ & 1 \\
\hline desconocido* & $13 \quad(8,7)$ & $32(10,7)$ & \\
\hline \multicolumn{4}{|l|}{ Ac. VIHA } \\
\hline positivo & $3(2,0)$ & $2(0,7)$ & \\
\hline negativo & $80(53,3)$ & $195(65,0)$ & $3,7(0,49-31,92)$ \\
\hline desconocido & $67(44,7)$ & $103(34,3)$ & 1 \\
\hline \multicolumn{4}{|l|}{ Ac. $\vee \mathrm{HB}$} \\
\hline positivo & $43(28,7)$ & $134(44,7)$ & $0,5(0,30-0.18)$ \\
\hline negativo & $74(49,3)$ & $112(37,3)$ & 1 \\
\hline desconocido & $33(22,0)$ & $54(18,0)$ & \\
\hline \multicolumn{4}{|l|}{ Ac.VHC } \\
\hline positivo & $11(7,3)$ & $129(43,0)$ & $0,1(0,04-0.18)$ \\
\hline negativo & $82(54,7)$ & $86(28,7)$ & 1 \\
\hline desconocido & $57(38,0)$ & $85(28,3)$ & \\
\hline \multicolumn{4}{|l|}{ Lúes (VDRL) } \\
\hline positivo & $3(2,0)$ & $18 \quad(6,0)$ & $0,3(0,071.21)$ \\
\hline negativo & $101(67,3)$ & $198(66,0)$ & 1 \\
\hline desconocido & $46(30,7)$ & $84(28,0)$ & \\
\hline \multicolumn{4}{|l|}{ VIH } \\
\hline positivo & $5(3,3)$ & $127(42,3)$ & $0,1(0,02-0,13)$ \\
\hline negativo & $115(76,7)$ & $141(47,0)$ & 1 \\
\hline desconocido & $30(20,0)$ & $32(10,7)$ & \\
\hline \multicolumn{4}{|l|}{ SIDA } \\
\hline sí & $1(0,7)$ & $15(5,0)$ & $0,1(0,01-0,93)$ \\
\hline no & $107(71,3)$ & $230(76,7)$ & \\
\hline desconocido & $42(28,0)$ & $55(18,3)$ & \\
\hline \multicolumn{4}{|l|}{$\mathrm{TB}$} \\
\hline sí & $3 \quad(2,0)$ & $28 \quad(9,3)$ & $0,2(0,05-0.70)$ \\
\hline no & $142(94,7)$ & $264(88,0)$ & \\
\hline desconocido & $5(5,5)$ & $8 \quad(2,7)$ & \\
\hline
\end{tabular}

* Intraalermorreacción de Mantoux.

Los desconocidos no se incluyen en el análisis.

VIH+, sida y TB, ni tampoco en cuanto a los consumos de alcohol y drogas. Sin embargo, en relación al consumo de drogas por vía parenteral, se observó menor riesgo en los que llevaban menos de cinco años de residencia en nuestro país ( $\mathrm{p}=0,04 ; \mathrm{OR}=0,3$; IC $\left.{ }_{95 \%} 0,07-1,09\right)$. Analizando la situación administrativa y el tiempo de estancia según la zona de procedencia de los emigrantes, se observó que los procedentes del continente asiático (Pakistán, China, Filipinas...) eran quienes en mayor proporción se encontraban en situación irregular $(81,8 \%)$, presentando al mismo tiempo el $72,7 \%$ de ellos estancias de más de cinco años en nuestro país.

En el análisis multivariado se realizaron distintas comparaciones mediante modelos 
de regresión logística condicional, tanto para identificar las características diferenciales entre casos y controles, como para determinar si existían diferencias entre los propios inmigrantes en función de su situación administrativa. La comparación entre autóctonos e inmigrantes se refleja en un modelo que evidenció que los inmigrantes extranjeros tendían a vivir menos en familia (OR=0,2; IC ${ }_{95 \%} 0,12-0,37$ ) $\mathrm{y}$ eran con menor frecuencia seropositivos al VIH (OR=0,2; IC $\left.{ }_{95 \%} 0,05-0,49\right)$ y UDVP (OR=0,2; IC $95 \% 0,10-0,41)$ (tabla 4$)$.

Al comparar a nivel multivariado a los inmigrantes en función de su situación administrativa (regularizada o no), destaca que los primeros eran de mayor edad $\left(\mathrm{OR}=1,1\right.$; IC $\left.{ }_{95 \%} 1,01-1,13\right)$, tendían a vivir más en familia $\left(\mathrm{OR}=2,7 ; \mathrm{IC}_{95 \%} 1,23-5,80\right) \mathrm{y}$ presentaban un mayor consumo de alcohol (OR=1,7; IC $95 \%$ 1,07-2,59) (tabla 5).

\section{DISCUSIÓN}

El principal objetivo de este estudio era determinar si algunos indicadores salud de los inmigrantes extranjeros procedentes de zonas de bajo desarrollo social y económico, identificados en un entorno penitenciario, diferían de los detectados en la población autóctona. Para este propósito, se seleccionó el $\mathrm{CPHB}$, dado que en él se hallan algunos de los sectores de nuestra población con más factores de riesgo social y económico frente a enfermedades como el sida, la TB y el consumo de drogas, toda vez que una elevada proporción de los internos, son inmigrantes extranjeros procedentes de áreas socioeconómicamente deprimidas. Este hecho, aunque facilita la obtención de la muestra de inmigrantes y de una muestra de UDVP, no permite la extrapolación de los resultados y sus interpretaciones al conjunto de la población de inmigrantes extranjeros o al conjunto de UDVP, aunque probablemente los datos

Tabla 4

Características diferenciales de los inmigrantes extranjeros frente a los autóctonos. modelo de regresión logística condicional

\begin{tabular}{|lccc|}
\hline VARIABLE & OR & IC 95\% & VALOR P \\
\hline UDVP (sí/no) & 0,2 & $0,10-0,41$ & $<0,001$ \\
Convivencia en familia (sí/no) & 0,2 & $0,12-0,37$ & $<0,001$ \\
Infección por el VIH (sí/no) & 0,2 & $0,06-0,50$ & 0,001 \\
\hline
\end{tabular}

Las variables edad y su interacción con UDVP, consumo de cannabis, conducta de riesgo heterosexual y tuberculosis, no presentaron diferencias estadísticamente significativas entre ambos grupos. No se incluyeron en los modelos previos las variables con un elevado número de datos faltantes.

Tabla 5

Características de los inmigrantes según su situación administrativa (regular/irregular). Análisis de regresión logística

\begin{tabular}{|lccc|}
\hline VARIABLE & O.R. & IC 95\% & VALOR P \\
\hline Edad (menor/mayor)* & 1,1 & $1,01-1,13$ & 0,02 \\
Convivencia en familia (sí/no) & 2,7 & $1,23-5,80$ & 0,01 \\
Consumo de alcohol (sí/no) & 1,7 & $1,07-2,59$ & 0,02 \\
\hline
\end{tabular}

* Analizada como variable continua.

Las variables UDVP, nivel de conocimientos de castellano, tiempo de residencia en el estado español no se asociaron de forma estadísticamente significativa con la variable dependiente. 
obtenidos sean similares a los de la numerosa población de estas características existente en nuestras instituciones penitenciarias. A pesar de ello, y en relación con este mismo aspecto, el que diversos cstudios cpidemiológicos hayan identificado los antecedentes de prisión como factor independiente asociado al riesgo de presentar $\operatorname{Sida}^{11,12} \mathrm{y}$ $\mathrm{TB}^{13,14}$, contribuía a considerar la idoneidad del entorno penitenciario para la realización de este estudio.

En relación al estudio de la TB en instituciones penitenciarias de otros países, se ha evidenciado, además de su elevada incidencia, la necesidad de llevar a cabo estrategias específicas de prevención y control, debido a las características propias de este tipo de entorno ${ }^{15.16}$, observándose, así mismo, una mayor prevalencia de trastornos de salud física y mental respecto a la población general $^{17}$.

Cabe destacar también, que en los países desarrollados se viene insistiendo en los últimos años en que la progresiva inmigración de personas procedentes de zonas socioeconómicamente menos favorecidas. incide directamente en el incremento de enfermedades infecciosas como la TB ${ }^{18.19}$. Este hecho, si bien ha sido puesto de manifiesto en zonas de baja incidencia de la enfermedad (Suiza, Holanda...), no ha sido demostrado en nuestro país, donde existen unas tasas de incidencia de $\mathrm{Tb}$ en la población autóctona residente en las áreas socioeconómicamente deprimidas, que se identifican más con las de los países de procedencia de estos inmigrantes que con las del mundo desarrolla$\mathrm{do}^{20}$. En este sentido, el análisis de los datos del Programa de Prevención y Control de la Tb de la ciudad de Barcelona, muestra como solamente el $7 \%$ del total de $\operatorname{casos}^{21}$ detectados anualmente, corresponde a inmigrantes extranjeros, observándose una estabilidad en los últimos 7 años. Esta cifra se reduce aún más cuando consideramos exclusivamente a las personas procedentes de los países del llamado Tercer Mundo, existiendo además la posibilidad de que algunos de estos pacientes hayan adquirido la $\mathrm{Tb}$ en nuestro país por residir en áreas marginales hiperendémicas, por haber ingresado en algún centro penitenciario o por algún otro factor de riesgo.

De entre los resultados del estudio destaca el menor consumo de drogas por vía parenteral, así como el bajo número de pacientes con infección VIH o sida entre los inmigrantes, fenómeno que puede estar relacionado con la baja prevalencia de heroinómanos en los países más pobres y con determinadas características religiosas o culturales.

En cuanto a la relativa superior prevalencia de pacientes tuberculín positivos entre los inmigrantes extranjeros, a parte de reflejar la mayor endemia tuberculosa de muchos de sus países de origen, debe pensarse en un posible efecto de la vacunación con $\mathrm{BCG}$, más frecuente en muchas de estas zonas, a pesar de que tal como se comenta en el apartado de métodos, no pudiera valorarse en este estudio su impacto real en la fase de recogida de información. En un estudio reciente realizado en Barcelona a nivel extrahospitalario, destaca que la prevalencia de la positividad a la prueba de la tuberculina en inmigrantes extranjeros, era del $43 \%{ }^{22}$, próxima a la de la población de autóctonos de nuestro estudio, e inferior a la que identificamos entre los inmigrantes extranjeros. Ello se debe probablemente a que en el citado trabajo se consideró la positividad a partir de los $10 \mathrm{~mm}$ de induración, en relación con los criterios vacunales, mientras que en este trabajo se aplicó el criterio de mayor o igual a $5 \mathrm{~mm}$.

En relación a las hepatitis virales, se ha descrito su elevada prevalencia entre algunos de los grupos de inmigrantes objeto de este estudio. Así, en un reciente estudio realizado en medio hospitalario en inmigrantes africanos residentes en el estado español, se demostró que la prevalencia de marcadores de hepatitis B era superior al $30 \%{ }^{23}$. Esta cifra es similar a la identificada en nuestro estudio, en el que la mayor seroprevalencia 
observada a nivel bivariado entre los autóctonos - a pesar del elevado número de desconocidos- representaría también un indicador de mejor estado de salud de los inmigrantes, aunque ello deba explicarse por el elevado número de toxicómanos entre la población de autóctonos, infectados en su mayoría por estos virus. En relación al sida, el hecho de que sólo se haya identificado un caso entre los inmigrantes frente a los 15 detectados en la población local, muestra la menor afectación de los primeros, a pesar del reducido número de personas. En lo que al consumo de drogas se refiere, a pesar de que los autóctonos presenten mayores niveles de consumo, se observa como los inmigrantes acceden con menor frecuencia a los programas de tratamiento para drogodependientes. En ello puede influir la irregular situación administrativa de muchos de los inmigrantes con las consiguientes restricciones para a recibir estas prestaciones, así como su desconfianza general a cualquier contacto con un recurso público, por el temor a scr cxpulsados.

A partir de estos datos podría afirmarse que la población autóctona presentaría un mayor consumo de drogas por vía parenteral y superiores niveles de infección por el $\mathrm{VIH}$, mientras que los inmigrantes extranjeros, vivirían menos en familia, identificándose con el tiempo con las conductas de riesgo de los ambientes de marginalidad. Con ello podríamos asumir a priori que la población inmigrante identificada en el CPHB, presenta un perfil y unos estilos de vida aparentemente más sanos que los autóctonos de este entorno - muy afectados por el consumo de drogas ilegales-, hecho que coincidiría con lo descrito en relación a que los inmigrantes económicos son pcrsonas jóvenes y $\operatorname{sanas}^{24}$, siendo las malas condiciones socioeconómicas, el hacinamiento y la marginación, los elementos que ponen en riesgo su salud.

Se observó que no existía relación entre mayor tiempo de residencia y regularización administrativa, evidenciándose que in- dividuos con estancias incluso superiores a diez años se hallaban en situación irregular. A pesar de ello cabe considerar la posibilidad de que la población inmigrante del CPHB no fuera representativa, en este aspecto, del conjunto de la población inmigrada. Así, si bien los individuos con períodos de estancia más prolongados presentan una mayor estabilidad sociodemográfica identificada a partir de las reagrupaciones familiares, este hecho no se traduce en nuestro estudio en una mejora de su situación administrativa.

En cuanto a las limitaciones del presente trabajo, cabe destacar, en primer lugar, las propias características del centro penitenciario, que por tratarse de un recurso para internos preventivos, determina en muchos casos estancias muy cortas en algunos de los internos. Este hecho ha contribuido sin duda a que la cifra de casos en los que no se pudo disponer de información sobre aspectos como la serología frente al VIH, haya sido superior a la identificada en otros contextos. Para incrementar estas cifras cabría pensar en la implantación de estrategias específicas de cribaje frente al VIH, tal como se ha planteado en otros estudios ${ }^{25,26}$. En relación a la comparabilidad entre ambas poblaciones, las restricciones de la actual legislación española en materia de inmigración extranjera, determinan con frecuencia el ingreso en prisión de los inmigrantes por delitos derivados de su precaria situación económica y administrativa, mientras que en el caso de los autóctonos, los internamientos se asocian más a hechos delictivos relacionados con el consumo de drogas, lo que podría implicar un cierto sesgo de selección entre éstos últimos en relación a algunos de los parámetros estudiados (VIH, consumo de drogas...).Por otro lado, el hecho de que se trate de un estudio de prevalencia puntual, no permite disponer de datos de incidencia para lo que se requeriría llevar a cabo un estudio de seguimiento, de difícil realización en una prisión preventiva como el CPHB, aunque planteable en otro contexto. 
Para concluir, resaltamos que los inmigrantes extranjeros identificados en el CPHB presentan, en relación al sida, la $\mathrm{Tb}$, las hepatitis víricas y el consumo de drogas, una situación más favorable que la de sus controles autóctonos, aunque las dificultades a que deben enfrentarse después de haber emigrado ${ }^{27}$, determinan para muchos de ellos el camino hacia la marginalidad con el consiguiente incremento de riesgos para su salud. Por todo ello, es necesario insistir en la normalización de la situación administrativa de estos inmigrantes que disponen de un buen estado de salud inicial y una voluntad de desenvolverse en nuestro entorno ${ }^{24,28}$. En caso contrario, los problemas económicos, sociales y administrativos, podrían contribuir a incrementar la problemática de las toxicomanías y/o la marginalidad, que constituye el factor clave de los ingresos en las instituciones penitenciarias de nuestro país.

\section{BIBLIOGRAFÍ $\Lambda$}

1. Urenda JI, Informe sobre immigració estrangera a Barcelona. Barcelona: Oficina del Comissionat de l'Alcaldia per a la defensa dels Drets Civils; 1993.

2. Observatori permanent de la immigració a Barcelona. Dades del Govern Civil II. Barcelona: Ajuntament de Barcelona; 1996.

3. Pereda C, Prada MA, Actis W. Situación y problemática de los inmigrantes en España. Informe contextual 1991. Madrid: Cáritas española; 1991.

4. Ganduxer D, Villalbí JR. Asistencia médica y cobertura vacunal de los hijos de mujeres de origen Filipino. An Esp Pediatr 1993; 39:235-239.

5. Jansà JM, Caylà JA, Galdós H, García de Olalla $\mathrm{P}$, Plasència A. Tuberculosis in migrating populations en Barcelona, 28 th World Conference of IUATLD/UICTMR Maiml, Germany $14^{\text {th }} 17^{\text {th }}$ June 1994. Tuber Lung Dis (suplement) 1994; 75:72-73.

6. Borrell C, Jansà JM, Pasarín M, Plasència A. La salut dels Immigrants (informe 1997). Actualització de l'anàlisi de les estadístiques vitals a la ciutat de Barcelona. Observatori Permanent de la Immigració de Barcelona 1997.
7. García-Vidal. Informe del Consell asesor de Benestar Social. Població estrangera al CPHB. Bar celona: Generalitat de Catalunya; 1991.

8. Els estrangers a les presons de Catalunya 19901994. Barcelona: Centre d'Estudis Jurídics i Formació Especialitzada. Justidata 12; 1996.

9. McKenna MT, McCray, Onorato I. The epidemiology of tuberculosis among foreign-born persons in the United States, 1986 to 1993. N Engl J Med 1995; 322:1071-6.

10. Russell M, Welte JW, Grace MB. Quatity-frequency measures of alcohol consumption: beverage-specific vs global questions. $\mathrm{Br} \mathrm{J}$ Addiction 1991; 86: 409-417.

11. Marco A, Guerrero RA, Rodríguez AM, Escribano M, Humet V, Mercadé E et al. Características de los casos de SIDA detectados en una prisión de Barcelona (1991-1993). Aten Primaria 1995; 15: 487-490.

12. Brewer TF, Derrikson J. AIDS in prison: a review of epidemiology and preventive policy. AIDS 1992; 6:623-628.

13. Caylà JA, Marco A, Bedoya A, Guerrero F, Garcia $J$, Martín $V$ et al. Differential Characteristics of AIDS Patients with a History of Imprisonment. IJE 1995; 24:1188-96.

14. Bellin EY, Fletcher DD, Safyer SM. Association of Tuberculosis Infection With Increased Time in or Admission to the New York City Jail System. JAMA 1993; 2228-2231.

15. Safyer SM, Richmond L, Bellin E, Fletcher D. Tuberculosis in Correctional Facilities: The Tuberculosis Control Program of the Montefiore Medical Center Rikers Island Health Services. The Journal of Law, Medicine \& Ethics 1993; 21:342351.

16. Drobniewski F. Tuberculosis in prisons-forgotten plague. Lancet 1995; 346:948-949.

17. Berkman A. Prison Health: the breaking point.AJPH 1995; 85:1616-1618.

18. Zuber PL, Binkin NJ, Ignacio AC, Marshall KL, Tribble SP, Tipple MA et al. Tuberculosis Screening for Immigrants and Refugees, Diagnostic Outcomes in the State of Hawaii. Am J Respir Crit Care Med 1996; 154:151-5.

19. Zellweger JP. Screening for Tuberculosis in risk groups in Switzerland. Bulletin des Bundesamtes für Gesundheitswesen 1993; 41:739-743. 
20. Rey A, Ausina V, Casal M, Caylà J, De March $P$, Moreno S. Situación actual de la tuberculosis en España. Una perspectiva sanitaria en precario respecto a los paises desarrollados. Med Clin (Barc) 1995; 105:703-707.

21. Caylà JA, Galdós-Tangüis H, Jansà JM, García de Olalla P, Brugal T, Pañella H. Evolución de la tuberculosis en Barcelona (1987-1995). Influencia del Virus de la inmunodeficiencia Humana y de las medidas de control; (en prensa).

22. Durán E, Cabezos J, Ros M, Terré M, Zarzuela $\mathrm{F}$, Bada JL. Tuberculosis en inmigrantes recién llegados a Barcelona. Med Clin (Barc) 1996; 106:525-528.

23. Lopez-Vélez R. Enfermedades infecciosas en inmigrantes. Formación Médica Continuada (FMC) $1996 ; 4: 222-228$.
24. Bada JM, Bada RM. Repercusiones sociosanitarias de la inmigración en España. Formación Médica Continuada (FMC) 1996; 5:277-78.

25. Voluntary Testing for Human Immunodeficiency Virus (HIV) in a Prison Population with a High Prevalence of HIV. Behrendt C, Kending N, Dambita C, Horman J, Lawlor J et al. Am J Epidemiol 1994; 139:918-926.

26. Diamond J. HIV testing in prison: what's the controversy. Lancet $1994 ; 344: 1650-1651$.

27. De Balanzó X. Enfermedades importadas y medicina de la inmigración. Aten Primaria 1991; 8:527529.

28. Jansà JM, Villalbí JR. La Salud de los inmigrantes y la atención primaria. Aten Primaria 1995: $15: 320-327$. 